\title{
Medication Nonadherence Among Medicare Beneficiaries with Comorbid Chronic Conditions: Influence of Pharmacy Dispensing Channel
}

Reethi N. Iyengar, PhD, MBA, MHM; Abbey L. LeFrancois, PharmD; Rochelle R. Henderson, PhD, MPA; and Rebecca M. Rabbitt, MS, PharmD

\begin{abstract}
BACKGROUND: Taking medications as prescribed is imperative for their effectiveness. In populations such as Medicare, where two thirds of Medicare beneficiaries have at least 2 or more chronic conditions requiring treatment with medications and account for more than $90 \%$ of Medicare health care spend, examining ways to improve medication adherence in patients with comorbidities is warranted.
\end{abstract}

OBJECTIVE: To examine the association of pharmacy dispensing channel (home delivery or retail pharmacy) with medication adherence for Medicare patients taking medications with comorbid conditions of diabetes, hypertension, and high blood cholesterol (3 of the top 5 most prevalent conditions), while controlling for various confounders.

METHODS: A retrospective analysis was conducted using de-identified pharmacy claims data from a large national pharmacy benefits manager between 0ctober 2010 and December 2012. Continuously eligible Medicare Part D patients (Medicare Advantage Prescription Drug plan and Prescription Drug Plan only) aged 65 years or older who had an antidiabetic, antihypertensive, and antihyperlipidemic prescription claim between October and December 2010 were identified and analyzed over a 2-year period. Multivariate logistic regression was used to evaluate the association between dispensing channel (DC) and medication adherence in calendar year (CY) 2012 controlling for prior adherence behavior (adherence in CY2011), differences in demographics, low-income subsidy status, days supply, disease burden, and drug-use pattern. Patients with a proportion of days covered (PDC) of at least $80 \%$ for each of the 3 conditions were considered to be adherent, and patients with PDC less than $80 \%$ for each of the 3 conditions were considered to be nonadherent. Patients were assigned to a DC depending on where they filled at least $66.7 \%$ of their prescriptions for each of the 3 conditions, and the rest were assigned to a mixed channel group.

RESULTS: The final analytical sample consisted of 40,632 patients. The adjusted odds of adherence for patients using home delivery were 1.59 $(95 \% \mathrm{Cl}=1.40-1.80)$ higher compared with patients using retail channels to obtain their prescriptions.

CONCLUSIONS: Medicare Part D patients taking medications for comorbid conditions who used home delivery had a greater likelihood (adjusted) of adherence than patients who filled their antidiabetic, antihypertensive, and antihyperlipidemic prescriptions using retail channels. Managed care stakeholders looking to make informed decisions in a cost-constrained environment to assess, implement, and promote solutions that improve health outcomes should consider the use of home delivery of prescriptions to improve adherence for Medicare Part D patients with comorbid conditions.

J Manag Care Spec Pharm. 2016;22(5):550-60

Copyright $\odot 2016$, Academy of Managed Care Pharmacy. All rights reserved.

\section{What is already known about this subject}

Medicare beneficiaries with 2 or more chronic conditions account for more than $90 \%$ of Medicare health care spend in addition to having poor health outcomes and a greater degree of functional dependence.

Pharmacotherapy is a key factor in restricting disease progression and ameliorating health outcomes; however, medication nonadherence has been linked to poor health outcomes and increased health care spending.

Dispensing channel has been positively associated with adherence to medications for diabetes, hypertension, and high blood cholesterol when examined independently in the Medicare population.

\section{What this study adds}

Compared with retail 90-day supply, home delivery 90-day supply indicated a much stronger association with adherence among Medicare beneficiaries taking medications for comorbid conditions than previously published estimates for individual therapy classes among Medicare beneficiaries.

Increased likelihood of adherence among Medicare home delivery patients with comorbid conditions is not just an artifact of auto-refill programs, healthy adherer effect, lower proportion of patients on low-income subsidy, and increased days of supply. This study provides evidence that home delivery is an especially useful solution to the problem of adherence and provides the necessary logistical support to address medication nonadherence among Medicare patients with comorbid conditions who have a greater degree of functional dependence.

A mong Medicare beneficiaries, diabetes, hypertension, and high blood cholesterol are in the top 5 of most prevalent chronic conditions, and $29.9 \%$ of Medicare beneficiaries have all 3 of those conditions. ${ }^{1}$ The prevalence of these conditions is expected to worsen with the aging of the U.S. population, especially with baby boomers reaching the Medicare eligibility age of 65 at the rate of 10,000 a day. ${ }^{2}$ These chronic conditions constitute major risk factors for serious cardiovascular and cerebrovascular conditions and events such as coronary heart disease, chronic kidney disease, congestive heart failure, and stroke. Beneficiaries with multiple chronic 
conditions are more likely to be hospitalized; have more emergency room visits; require more postacute care services, home health visits, and doctor visits; and have greater hospital readmission rates compared with Medicare beneficiaries with 1 or no chronic condition. ${ }^{1}$ In addition to the clinical impact of having comorbid conditions, Medicare spending, on average, increases with the number of chronic conditions (for which there are no known cures but can be managed by taking medications) regardless of sociodemographic group. Patients with multiple chronic conditions account for almost $98 \%$ of hospital readmissions and more than $90 \%$ of Medicare health care spend. ${ }^{1}$

Although prescription medications play an essential role in limiting chronic disease progression and ameliorating health outcomes, ${ }^{3,4}$ adherence to these medications is suboptimal. Medication adherence (defined as taking medications as prescribed) is integral to the effectiveness of pharmacotherapy. Nonadherence to medications has been linked to poor health outcomes and decrease in survival likelihood. ${ }^{5}$ In addition to clinical outcome impact, poor adherence to medications is widely recognized as a significant contributor of waste, inefficiency, and financial drain on the health care system. ${ }^{6} \mathrm{~A}$ previously published study suggests extra health care spending of $\$ 49-\$ 840$ (Medicare costs) per beneficiary per month for medication nonadherence. ${ }^{7}$

The most common reasons for nonadherence for Medicare beneficiaries include forgetting to take medications, procrastinating on refills or renewals of prescriptions, and transportation problems or caregiver time dependencies. ${ }^{8-10}$ Literature suggests that patients' functional dependence grows as the number of chronic conditions increases, making access to prescription medications more difficult..11,12 Additionally, financial concerns may impede adherence. ${ }^{13}$ As Medicare patients exhaust their prescription benefits and enter the "coverage gap," patients are required to pay more, which may result in the reduction of the number of prescriptions they fill. ${ }^{10,14}$ Medicare patients who fill medications for 3 conditions simultaneously may reach the coverage gap sooner and be at a higher risk of cost-related nonadherence, compared with patients who have just 1 condition.

Published literature suggests that dispensing channel can play a key role in improving medication adherence, including in the Medicare population. ${ }^{15-18}$ Several studies have indicated that obtaining medication through home delivery is 1 of many ways to address nonadherence. ${ }^{15,16}$ Home delivery pharmacies, in general, have been promoted as more cost-effective, convenient, and associated with fewer preventable emergency room visits in patients aged 65 years or older, compared with retail pharmacies. ${ }^{18-20}$ In contrast, proponents of retail pharmacies promote the benefits of pharmacist face-to-face interaction with patients. ${ }^{21,22}$ Previous literature suggests a positive relationship between functional dependence and chronic diseases among the elderly. Specifically, coexisting conditions increases the percentage of patients with disability, with nearly $25 \%$ of patients with chronic conditions reporting limitations in functional activity. ${ }^{23,24}$ In cases of patients with comorbidities and increased functional limitation, home delivery may provide medications in a convenient manner thereby avoiding nonadherence.

Previous studies on dispensing channel and medication adherence have examined therapy classes independently., ${ }^{910}$ To our knowledge, this study is the first to examine the association of dispensing channel with medication adherence among Medicare beneficiaries with comorbid conditions. The objective of this study was to examine the effect of dispensing channel on adherence to medications among Medicare beneficiaries who are taking medications for these 3 conditions: diabetes, hypertension, and high blood cholesterol, controlling for other known confounders. These confounders include the "healthy adherer effect" (e.g., a beneficiary's predisposition to engage in healthy alternatives), ${ }^{25,26}$ home delivery auto-refill program impact, and differences in days supply between home delivery and retail pharmacies. We hypothesized that, compared with retail, the home delivery channel is associated with better adherence rates among Medicare beneficiaries with comorbid conditions, similar to the association found in previous studies that examined adherence to therapy classes independently., ${ }^{9}$

\section{Methods}

\section{Study Population and Research Design}

This study used prescription claims data from Medicare Part D beneficiaries (low-income subsidy and no low-income subsidy patients) whose pharmacy benefits were managed by a large national pharmacy benefit management company. Inclusion was limited to patients who were continuously enrolled with pharmacy benefits from October 1, 2010, to December 31, 2012; who were aged 65 years or older; and who had at least 1 prescription claim during the index period of October 1, 2010, to December 31, 2010 for each of these medication groups: antidiabetics (oral), antihypertensives, and antihyperlipidemics. Patients who were enrolled in a home delivery auto-refill program during the main analytical period were excluded from the study.

A retrospective claims analysis was conducted over a 3-year period that was divided into 4 distinct phases (Figure 1). The index period, October 1, 2010-December 31, 2010, was used to identify Medicare beneficiaries to be included in the study. Published literature suggests that previous adherence could be indicative of health-conscious behaviors and a measure of better health-seeking tendency. ${ }^{27}$ A patient's previous adherence was used as a proxy measure to control for self-selection bias because of the prior adherence behavior (PAB) effect in the multivariate model. Previous adherence was based on the patient's proportion of days covered (PDC) in the year 2011 irrespective of the dispensing channel, which was considered 
as the baseline period. Calendar year (CY) 2012 constituted the main analysis period for this study. In addition to the index period, patients needed to have at least 1 claim in each of the 3 therapy classes in all distinct periods of the study timeline.

A 2-year design was selected in order to have 1 full year (CY2011) to calculate PAB and 1 full year (CY2012) as the analysis period. The timeline was intentionally selected to ensure that PAB was calculated immediately preceding the main analysis period. Figure 1 presents the study timeline. Figure 2 presents the sample selection algorithm with each of the exclusion criteria listed and percentage of patients retained.

Under provisions of the Health Insurance Portability and Accountability Act of 1996 (HIPAA) ${ }^{28}$ all individual patient identifiers were removed to maintain the privacy of protected health information from analytical datasets. The design of this study was not submitted to an institutional review board , since only de-identified administrative data were used..$^{19,29}$ All prescription claims were adjusted to 30-day equivalents by dividing the days supply by $30 ., 9$

\section{Study Variables}

The primary dependent variable was patient adherence to diabetes, hypertension, and high blood cholesterol medications, defined as a PDC of $80 \%$ or greater for each of the 3 conditions in the analysis period. ${ }^{30,31}$ Patients with PDC less than $80 \%$ for each of the 3 conditions were considered nonadherent. Hence, patients had to be adherent in all 3 therapy classes to be considered adherent. Patients were considered nonadherent if they were nonadherent to even 1 of the therapy classes. Patients' PDCs were first calculated at the drug group level-the combination of 8-digit Generic Product Identifiers (GPI) codes and clinically appropriate drug groups-by taking the total days supply divided by 365 days and multiplying the result by 100 , capping it at $100 \%$. Next, each drug group within the therapy classes was averaged to the therapy class level for each patient (Appendix A, available in online article) to calculate the PDC for the patient and the disease. Drug groups were based on a proprietary method of classifying medications into clinically accepted drug subclasses within each therapy class and maintained by a panel of clinical experts. Only the numbers of medication units actually meant to be taken during the study periods were included in the calculations. Spill-ins, defined as the parts of any claim that were in possession before the key periods (2011 for baseline and 2012 for analysis), and spillouts, defined as any excess in possession after the ends of the periods, were excluded from adherence calculations. Spill-ins and spill-outs were accounted for in the PDC calculations.

In addition to dispensing channel, other patient characteristics included in this study were age, gender, patient out-ofpocket (OOP) costs for 30-day adjusted prescriptions for each of the 3 conditions, location (urbanicity) of the patient, lowincome subsidy (LIS) status, average days supply per claim for each of the 3 conditions, previous adherence for each of the 3 conditions, disease burden, and severity of illness for diabetes and hypertension.

Channel determination was based on where the patient obtained at least two thirds (66.7\%) of their 30-day adjusted prescriptions in the year 2012. Those who did not receive at least $66.7 \%$ of their 30 -day adjusted prescriptions from either home delivery or retail were assigned to a mixed channel group. Age was calculated as the age of the patient on December 31, 2010. OOP costs for 30-day adjusted prescriptions were calculated by dividing total OOP pharmacy costs (for each therapeutic class) for the patient in 2012 by that patient's total number of 30-day adjusted prescriptions. The location variable (urbanicity) was based on the "core based statistical area" used by the U.S. Census Bureau to ascertain the urban core of metropolitan and micropolitan statistical areas.

Patient LIS status was also controlled for in the model as a proxy for socioeconomic status. Low-income subsidies are provided to low-income Medicare beneficiaries to provide assistance with the cost of drug coverage. Inclusion was limited to patients who remained exclusively on 1 status (either LIS or not) during the main analysis period (CY2012).

In order to address the suggestion that better adherence in home delivery is an artifact of greater days supply, average days supply per claim for each of the 3 conditions were used as a proxy measure to control for the differences in days supply of prescriptions between retail and home delivery channels. PAB was used as a proxy indicator for the healthy adherer effect, since past behavior has been known to predict future behavior. A similar method was used in a 2013 study that examined the association of dispensing channel with medication adherence to control for the healthy adherer effect for commercially insured patients and in a 2014 study that examined the same association among Medicare beneficiaries with just 1 
Medication Nonadherence Among Medicare Beneficiaries with

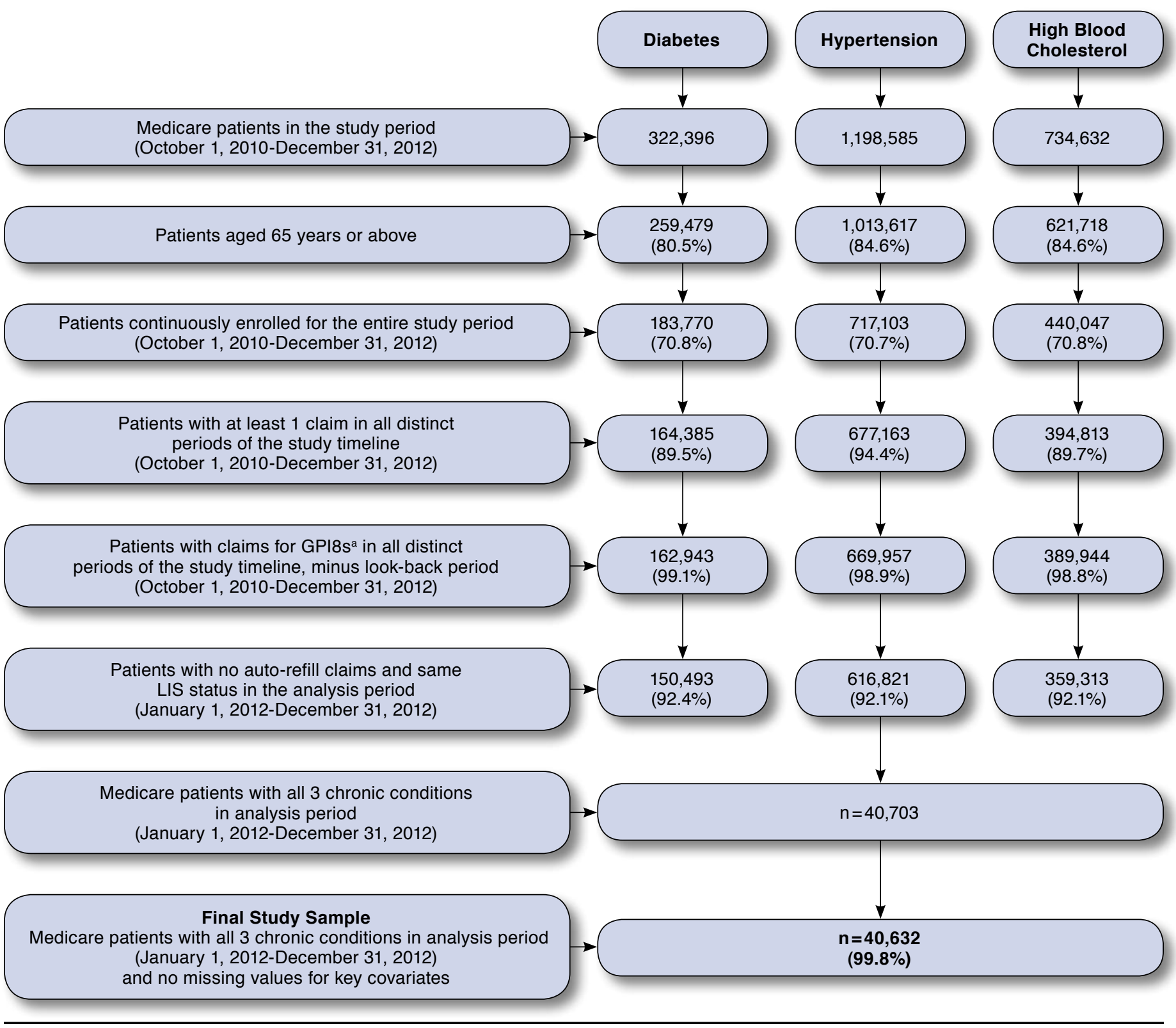

${ }^{a}$ GPI8s used in this study are listed in Appendix A, available in online article. GPI8=8-digit Generic Product Identifier; LIS=low-income subsidy.

chronic condition., ${ }^{9} 10$ Patient-level PDC was calculated for the 2010 time period to establish previous adherence. Medicare patients with PDC $\geq 80.0 \%$ during 2011 were classified as being adherent in the previous period.

In addition to sociodemographic variables in this study, proxy covariates controlled for patient disease burden and severity of illness. Patient overall disease burden was defined as the number of unique 2-digit GPIs (except diabetes, hypertension, and high blood cholesterol), which indicated distinct drug therapy classes used by patients beyond the 3 therapy classes. Number of unique drug groups for which patients had prescription claims in 2012 defined severity of illness for diabetes and hypertension. For high blood cholesterol, only adherence to statins was analyzed and so involved only 1 
TABLE 1 Baseline Characteristics Across Channels

\begin{tabular}{|c|c|c|c|c|c|c|}
\hline & \multicolumn{2}{|c|}{ Home Delivery } & \multicolumn{2}{|c|}{ Retail } & \multicolumn{2}{|c|}{ Mixed } \\
\hline \multirow[t]{2}{*}{ Number } & \multicolumn{2}{|c|}{5,624} & \multicolumn{2}{|c|}{33,338} & \multicolumn{2}{|c|}{1,741} \\
\hline & \multicolumn{6}{|c|}{ Mean (SD) } \\
\hline Age, years & 73.7 & (6.3) & 73.7 & (6.3) & 73.9 & $(6.2)$ \\
\hline OOP costs per 30-day adjusted prescriptions for antidiabetics & 10.53 & $(14.20)^{\mathrm{a}}$ & 6.39 & $(13.16)^{\mathrm{b}}$ & 10.61 & $(15.28)^{\mathrm{a}}$ \\
\hline OOP costs per 30-day adjusted prescriptions for antihypertensives & 7.60 & $(8.36)^{\mathrm{b}}$ & 4.42 & $(6.72)^{\mathrm{b}}$ & 7.83 & $(8.11)^{b}$ \\
\hline OOP costs per 30-day adjusted prescriptions for antihyperlipidemics & 10.87 & $(13.45)^{\mathrm{b}}$ & 5.84 & $(10.98)^{\mathrm{b}}$ & 11.21 & $(14.54)^{b}$ \\
\hline Disease burden proxyc & 2.27 & $(2.74)^{\mathrm{b}}$ & 5.43 & $(4.81)^{\mathrm{b}}$ & 3.05 & $(3.63)^{\mathrm{b}}$ \\
\hline Severity of illness proxy for diabetes ${ }^{\mathrm{d}}$ & 1.51 & $(0.68)$ & 1.50 & $(0.67)$ & 1.54 & $(0.68)^{\mathrm{b}}$ \\
\hline Severity of illness proxy for hypertension ${ }^{\mathrm{d}}$ & 2.17 & $(0.94)^{\mathrm{b}}$ & 2.24 & $(0.95)^{\mathrm{e}}$ & 2.25 & $(0.92)^{\mathrm{e}}$ \\
\hline Average days supply per claim for antidiabetics & 87.64 & $(7.40)^{\mathrm{b}}$ & 46.03 & $(24.32)^{\mathrm{b}}$ & 75.77 & $(21.10)^{\mathrm{b}}$ \\
\hline Average days supply per claim for antihypertensives & 87.17 & $(7.37)^{\mathrm{b}}$ & 46.24 & $(23.78)^{b}$ & 75.47 & $(19.42)^{\mathrm{b}}$ \\
\hline \multirow[t]{2}{*}{ Average days supply per claim for antihyperlipidemics } & 88.36 & $(6.13)^{\mathrm{b}}$ & 47.32 & $(25.62)^{b}$ & 80.07 & $(18.97)^{\mathrm{b}}$ \\
\hline & \multicolumn{6}{|c|}{ n (\%) } \\
\hline Baseline adherent for antidiabetics & 4,548 & $(80.9)^{b}$ & 21,097 & $(63.3)^{\mathrm{b}}$ & 1,160 & $(66.7)^{\mathrm{b}}$ \\
\hline Baseline adherent for antihypertensives & 4,688 & $(83.4)^{b}$ & 21,336 & $(64.0)^{\mathrm{b}}$ & 1,217 & $(69.9)^{\mathrm{b}}$ \\
\hline Baseline adherent for antihyperlipidemics & 5,033 & $(89.5)^{\mathrm{b}}$ & 23,677 & $(71.1)^{\mathrm{b}}$ & 1,409 & $(81.1)^{\mathrm{b}}$ \\
\hline Female & 2,500 & $(44.5)^{\mathrm{b}}$ & 19,672 & $(59.0)^{\mathrm{b}}$ & 901 & $(51.8)^{\mathrm{b}}$ \\
\hline Urbanicity & 5,302 & $(94.3)$ & 31,641 & $(94.9)$ & 1,677 & $(96.3)^{\mathrm{b}}$ \\
\hline LIS beneficiaries & 206 & $(3.7)^{\mathrm{b}}$ & 11,740 & $(35.2)^{\mathrm{b}}$ & 104 & $(6.0)^{\mathrm{b}}$ \\
\hline $\begin{array}{l}\text { aSignificantly different at } P<0.05 \text { from retail channels only. } \\
\text { bSignificantly different at } P<0.05 \text { from both other groups. } \\
\text { cPatient overall disease burden was defined as the number of unique 2-digit } \\
\text { therapy classes used by the patient beyond the } 3 \text { therapy classes. } \\
\text { dSeverity of illness is defined by the number of unique drug groups for which } \\
\text { eSignificantly different at } P<0.05 \text { from home delivery only. } \\
\text { GPI=Generic Product Identifer; LIS=low-income subsidy; OOP = out of poc }\end{array}$ & $\begin{array}{l}\text { xcept did } \\
\text { ient had } \\
=\text { standa }\end{array}$ & $\begin{array}{l}\text { es, hyperte } \\
\text { rescription } \\
\text { leviation. }\end{array}$ & $\begin{array}{l}\text { and high b } \\
\text { n } 2012 \text { fo }\end{array}$ & d cholester & nsion. & distinct dru \\
\hline
\end{tabular}

drug group. Percent adherent was defined as the percentage of patients in each cohort (home delivery/retail/mixed) who had a PDC of $80 \%$ or greater for each of the 3 conditions.

\section{Analysis}

From a sample of more than 4 million Medicare beneficiaries, 322,396 diabetes patients, 1,198,585 hypertension patients, and 734,632 high blood cholesterol patients were identified as meeting the study requirements between October and December 2010. Figure 2 presents the sample selection algorithm for the study, which resulted in a final analytical sample of data for 40,632 patients who were continually treated pharmacologically for each condition (diabetes, hypertension, and high blood cholesterol) throughout the entire study period.

Descriptive statistics were estimated, and bivariate differences between groups were tested using Kruskal-Wallis (nonparametric) tests for all continuous variables and chi-square tests for categorical variables. Multivariate logistic regression analyses were performed to estimate the association of dispensing channel with the odds of being adherent, controlling for the aforementioned confounders.

Additionally, an analysis of a subsample of patients filling exclusively 90-day prescriptions evaluated the impact of channel on adherence. Inclusion was limited to patients who filled all of their prescriptions through 1 channel (either retail or home delivery). The final analytical subanalysis sample constituted data for 4,157 patients. All analyses were conducted using SAS version 9.3 (SAS Institute, Cary, NC).

\section{Results}

\section{Descriptive Findings}

Patients using home delivery tended to be similar in age to those using retail and mixed channels but included a lower proportion of females than either retail or mixed channels (Table 1). Home delivery patients had higher average days supply per claim, lower average disease burden, and similar mean severity of illness for diabetes but slightly lower mean severity of illness for hypertension compared with retail patients. Home delivery patients also had slightly higher OOP per 30-day adjusted prescriptions than retail patients, an artifact of drug-mix (branded products vs. generics differences between dispensing channels) and differences in proportion of beneficiaries with LIS. Proportion of patients with LIS status was significantly lower in the home delivery group compared with the retail and mixed groups. Across all 3 classes, previous adherence was significantly higher in the home delivery channel than in the retail or mixed channels, reflecting the importance of using a proxy measure to control for PAB in the 


\begin{tabular}{|c|c|c|c|c|c|c|c|c|c}
\hline \multirow{2}{*}{} & \multicolumn{2}{|c|}{} & \multicolumn{2}{|c|}{} & \multicolumn{2}{|c|}{$\begin{array}{c}\text { Difference Between } \\
\text { Home Delivery } \\
\text { and Retail }\end{array}$} & $\begin{array}{c}\text { Difference Between } \\
\text { Home Delivery } \\
\text { and Mixed }\end{array}$ \\
\hline All & 90 Days & All & 90 Days & All & 90 Days & All & 90 Days & All & 90 Days \\
\hline $91.3 \%$ & $94.7 \%$ & $68.3 \%$ & $89.4 \%$ & $72.3 \%$ & NA & 23.0 & 5.3 & 19.0 & NA \\
\hline
\end{tabular}

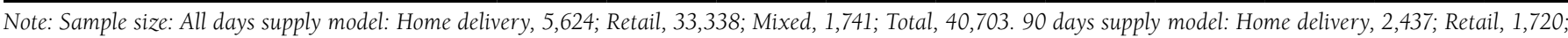
Total, 4,157.

NA = not applicable.

model in order to obtain less biased estimates of the effect of dispensing channel on adherence.

Unadjusted adherence rates for those using home delivery were consistently higher than for patients using retail. Home delivery had 23.0\% more adherent patients than retail (91.3\% vs. $68.3 \% ; P<0.001)$ and $19.0 \%$ more than mixed channels (91.3\% vs. $72.3 \% ; P<0.001)$, as presented in Table 2.

The results of a direct comparison for a subset of patients filling 90-day prescriptions $(n=4,157)$ in retail with patients filling 90-day prescriptions in home delivery also indicated similar results. Home delivery had $5.3 \%$ more adherent patients than retail (94.7\% vs $89.4 \% ; P<0.001$ ). Baseline characteristics of Medicare beneficiaries analyzed in this model are presented in Appendix B (available in online article).

\section{Multivariate Findings}

The significance of controlling for the PAB effect and the impact of channel classification criterion and differential days supply are presented in Table 3. The findings offer insight into a more direct comparison between 90-day retail and 90-day home delivery, although only a small percentage of beneficiaries received 90-day supplies through retail. In Table 3, the Model 1 column presents the odds of patient (including all days supply and any dispensing channel) adherence, controlling for previous adherence behavior. Results of channel-adherence relationship for 90-day home delivery versus 90-day retail, controlling for the PAB effect, are presented in the Model 2 column. PAB was the strongest contributor to the odds of patient adherence. Odds ratios (ORs) ranged from 3.22 to 5.74 , indicating that adherent beneficiaries in the baseline period were 3 to nearly 6 times more likely to be adherent in the main analysis period irrespective of the channel from which they filled their medications.

After controlling for relevant population differences (age, gender, and patient OOP cost per 30-day adjusted prescriptions) and key covariates ( $\mathrm{PAB}$, average days supply per claim, disease burden, severity of illness, and urbanicity), the differences in adherence between the dispensing channels $(\mathrm{OR}=1.59,95 \%$ confidence interval $[\mathrm{CI}]=1.40-1.80)$ is significant (Table 3, Model 1). In 2012, the odds of a home delivery patient with comorbid conditions being adherent was $59 \%$ higher than a patient using the retail channel for diabetes, hypertension, and high blood cholesterol medications. The odds of being adherent for a patient using mixed channels was $38 \%$ lower than patients using the retail channel.

Adjusted adherence rates for those using home delivery was higher than for patients using retail (Table 4). Home delivery had $6.8 \%$ more adherent patients than retail and $15.9 \%$ more than those using mixed channels.

Additional sensitivity analysis examining patients filling only 90-day prescriptions (Table 3, Model 2) concluded that the adjusted odds of being adherent were significantly higher in home delivery compared with retail $(\mathrm{OR}=1.95,95 \% \mathrm{CI}=1.46$ 2.59), indicating that home delivery patients with comorbid conditions of diabetes, hypertension, and high blood cholesterol were 95\% more likely to be adherent than their respective retail counterparts, even after equalizing the days supply differences and addressing the channel attribution cutoff.

\section{Discussion}

The descriptive and multivariate findings from this study provide support for the study hypotheses. Medicare beneficiaries with comorbid conditions of diabetes, hypertension, and high blood cholesterol using home delivery were significantly more adherent compared with their retail counterparts after controlling for demographics, drug use patterns, differences in days supply, and the PAB effect. Additionally, the results from the comorbid cohort were larger in magnitude in comparison with the association among Medicare beneficiaries for individual therapy classes, as presented in previously published literature. ${ }^{10}$ The findings from the main model, as well as the model comparing 90-day retail with 90-day home delivery users, were consistent. Also, the magnitude of the findings was amplified in the direct comparison model that compared 90-day retail with 90-day home delivery users, even after controlling for differences in patient characteristics such as comorbidity profile and severity of illness. The results strongly corroborate that home delivery leads to greater odds of adherence in Medicare patients with comorbid conditions even after accounting for differences in the PAB effect, adjusting for the difference in days supply in all models, and including only those patients who did not participate in a home delivery auto-refill program. 


\begin{tabular}{|c|c|c|c|c|}
\hline & \multicolumn{2}{|c|}{ Model $1(n=40,632)^{a}$} & \multicolumn{2}{|c|}{ Model $2(n=4,157)^{b}$} \\
\hline & Odds Ratio & $95 \% \mathrm{CI}$ & Odds Ratio & $95 \% \mathrm{CI}$ \\
\hline Home delivery vs. retail & $1.591^{\mathrm{c}}$ & $1.403-1.804$ & $1.946^{\mathrm{c}}$ & $1.461-2.593$ \\
\hline Mixed vs. retail & $0.619^{c}$ & $0.538-0.712$ & NA & NA \\
\hline Age & $0.980^{\mathrm{c}}$ & $0.975-0.984$ & 0.989 & $0.968-1.011$ \\
\hline Female vs. male & 0.981 & $0.925-1.041$ & 0.832 & $0.639-1.083$ \\
\hline Urbanicity & 1.026 & $0.900-1.169$ & 0.623 & $0.305-1.273$ \\
\hline Disease burden & $1.013^{\mathrm{c}}$ & $1.006-1.020$ & $1.073^{d}$ & $1.019-1.130$ \\
\hline LIS & 1.055 & $0.983-1.131$ & 0.863 & $0.554-1.344$ \\
\hline OOP costs 30-day adjusted for antidiabetics & $0.996^{\mathrm{d}}$ & $0.994-0.999$ & 1.000 & $0.989-1.011$ \\
\hline OOP costs 30-day adjusted for antihypertensives & 1.001 & $0.996-1.005$ & 1.004 & $0.985-1.024$ \\
\hline OOP costs 30-day adjusted for antihyperlipidemics & $0.995^{\mathrm{c}}$ & $0.992-0.997$ & 1.006 & $0.993-1.020$ \\
\hline Severity of illness for diabetes & $0.713^{c}$ & $0.683-0.743$ & 0.828 & $0.675-1.016$ \\
\hline Severity of illness for hypertension & $0.800^{\mathrm{c}}$ & $0.775-0.825$ & 0.895 & $0.766-1.046$ \\
\hline Prior adherence for antidiabetics & $3.222^{c}$ & $3.037-3.418$ & $3.685^{c}$ & $2.775-4.895$ \\
\hline Prior adherence for antihypertensives & $3.356^{c}$ & $3.164-3.561$ & $3.782^{c}$ & $2.820-5.073$ \\
\hline Prior adherence for antihyperlipidemics & $5.735^{c}$ & $5.387-6.106$ & $4.041^{\mathrm{c}}$ & $2.981-5.479$ \\
\hline Average days supply per claim for antidiabetics & $1.004^{d}$ & $1.001-1.006$ & NA & NA \\
\hline Average days supply per claim for antihypertensives & $1.011^{\mathrm{c}}$ & $1.009-1.013$ & NA & NA \\
\hline Average days supply per claim for antihyperlipidemics & 1.001 & $0.999-1.003$ & $\mathrm{NA}$ & $\mathrm{NA}$ \\
\hline \multicolumn{5}{|c|}{$\begin{array}{l}{ }^{b} \text { Model } 2 \text { presents the results of the comparison of 90-day retail with 90-day home delivery for patients filling their prescriptions exclusively through } 1 \text { channel (either hom } \\
\text { delivery or retail). }\end{array}$} \\
\hline
\end{tabular}

The multivariate adjusted coefficients on OOP costs, disease burden, and severity of illness are consistent with previous research. ${ }^{10}$ The literature suggests the need to address the confounding impact of patients' predisposition to be adherent while examining any adherence-related relationship. ${ }^{26,27,33}$ To our knowledge, this research is the first to address this concern among Medicare beneficiaries with comorbidities and so is less likely to be biased, since the confounding effect of patient predisposition to be adherent is accounted for in this study.

Home delivery pharmacies offer convenience to patients and are an effective alternative to retail pharmacies for continued access to medications. Access issues and financial difficulties that are identified as impediments to adherence are eased for many patients because prescriptions are delivered to the patient's home and the total patient OOP costs are frequently less in home delivery pharmacies. Higher financial burden because of multiple health conditions, as well as higher functional dependency issues among Medicare patients with comorbidities, could be driving higher adherence rates among home delivery patients in this study. Additionally, when patients switch channels frequently, they may not be able to benefit from programs and services (offered by retail or home delivery pharmacies) intended to improve adherence. This could be driving lower adherence rates in the mixed channels group compared with patients using home delivery or retail channels.
Other published studies suggest that improved adherence among beneficiaries with multiple chronic conditions can also considerably help reduce hospitalizations, emergency room visits, use of postacute care services, home health visits, doctor visits, and hospital readmission rates, which are known to be worse for patients with multiple conditions compared with those who have just 1 condition. In addition to positive clinical outcomes, the findings of this study have important financial implications for Medicare Advantage health plans apart from the clinical benefits (i.e., improved health outcomes) of better adherence. ${ }^{34}$ Better adherence may improve a Medicare health plan's star ratings, which could translate to greater Medicare reimbursement. ${ }^{35}$ Patients with multiple chronic conditions account for more than $90 \%$ of Medicare health care spend because of increased emergency room visits and hospitalizations partly because of nonadherence to their pharmacotherapeutic regimens. For patients with comorbid conditions of diabetes, hypertension, and high blood cholesterol, the average per capita Medicare spending equaled $\$ 17,451$ in $2010 .{ }^{1}$ By promoting the use of a more effective dispensing channel to address nonadherence among these patients, given the findings in the literature, Medicare may be able to reduce its health care spending, especially in the coming years when more and more baby boomers with these comorbid conditions are expected to become Medicare eligible. 


\begin{tabular}{|c|c|c|c|c|c|c|c|c|c|c|}
\hline & \multicolumn{2}{|c|}{ Home Delivery } & \multicolumn{2}{|c|}{ Retail } & \multicolumn{2}{|c|}{ Mixed } & \multicolumn{2}{|c|}{$\begin{array}{c}\text { Difference Between } \\
\text { Home Delivery } \\
\text { and Retail }\end{array}$} & \multicolumn{2}{|c|}{$\begin{array}{c}\text { Difference Between } \\
\text { Home Delivery } \\
\text { and Mixed }\end{array}$} \\
\hline & All & 90 Days & All & 90 Days & All & 90Days & All & 90 Days & All & 90 Days \\
\hline $\begin{array}{l}\text { Beneficiaries with comorbid conditions } \\
\text { (diabetes+hyperten-sion +high blood cholesterol) }\end{array}$ & $85.4 \%$ & $96.5 \%$ & $78.7 \%$ & $93.5 \%$ & $69.5 \%$ & NA & 6.8 & 3.1 & 15.9 & NA \\
\hline
\end{tabular}

Note: Sample size = All days supply model: Home delivery, 5,618; Retail, 33,280; Mixed, 1,734; Total, 40,632. 90 days supply model: Home delivery, 2,437; Retail, 1,720. NA = not applicable

\section{Limitations}

The findings from this study should be interpreted in view of its limitations. First, because the study sample was limited to prescription claim analysis for Medicare (Medicare Advantage Prescription Drug plan and Prescription Drug Plan only) patients aged 65 years and above, the results of the analyses may not be generalizable to other populations, such as dual eligible or Employer Group Waiver Plan beneficiaries. Second, the study used PDC as a proxy for medication-taking behavior, which assumes that a pill in hand is a pill taken but may not necessarily be true. However, the use of pharmacy claims to ascertain adherence to medications use has been well documented in previous studies. ${ }^{9,19,25,30,35}$ Further, the distinction between nonadherence and prescriber-recommended discontinuation or update in directions for use could not be made based on claims data.

Third, our study design included patients on medication for at least 2 years. Thus, patients who did not fill at least 1 prescription in each of the distinct time periods of this study (with the exception of the 6-month period before the index claim for each of the 3 therapy classes) were excluded from the analysis. However, within the Medicare population, diabetes, hypertension, and high blood cholesterol medications are used chronically, so one would expect the impact of this limitation to be minimal. Fourth, our results do not account for the differential impact of the coverage gap on medication adherence. Beneficiaries who were adherent to their medications before reaching the coverage gap might become nonadherent after reaching the gap. However, since Medicare patients who were being treated for all 3 conditions would reach the coverage gap sooner than Medicare patients being treated for just 1 condition, the limitation would equally affect both dispensing channels.

Fifth, we could not identify patients engaged in retail autorefill programs, so our estimates of retail adherence might be inflated and our study conclusions conservative. Sixth, the findings from our study are limited by the reliability and validity of the proxy measures used to control for the confounding effects of covariates in the relationship between dispensing channel and adherence. This study had access to only phar- maceutical claims data, so lack of medical information is a limitation of this study. Other studies should incorporate medical data when available to strengthen and validate the proxy variables, as well as provide robust results to underscore the relationship between dispensing channel and adherence. Seventh, patients using injectables for diabetes are excluded from this study. The results of this study are generalizable to patients using oral medications alone. Eighth, this study did not account for patients' functional dependence, which has been shown to be associated with adherence behavior in the literature because of data unavailability. ${ }^{36}$ Future studies should include this important factor when available.

Ninth, \$4 prescription programs introduced by chain pharmacies, which are not captured by prescription claims data, could play a role in lower observed adherence rates in retail pharmacies. Because of data limitations, these programs could not be accounted for in this study. However, given the magnitude of difference in adherence rates between retail and home delivery pharmacies, it is unlikely that all of it can be explained by $\$ 4$ prescription programs. Tenth, patients lost due to mortality, which cannot be identified using prescription claims data, could potentially confound the generalizability of the results. However, mortality rates should be similar between the 2 dispensing channels so as not to bias the findings. Last, the results of this study are generalizable to the Medicare population having comorbid conditions of diabetes, hypertension, and high blood cholesterol. Further studies are needed to establish the generalizability of these findings for adherence conclusions on other comorbid conditions.

\section{Conclusions}

The findings from this study indicate that Medicare patients with comorbid conditions receiving their medications via home delivery had greater adherence than those receiving the medications in retail pharmacies, even after accounting for differences in factors such as days supply and LIS status. By excluding beneficiaries who obtained their prescriptions via home delivery auto-refill programs, this study provides strong support that home delivery signifies an important alternative to help patients reach optimal adherence compared with retail pharmacies. The main model and the 90-day comparison 


\section{Medication Nonadherence Among Medicare Beneficiaries with \\ Comorbid Chronic Conditions: Influence of Pharmacy Dispensing Channel}

indicate that the home delivery channel is associated with improved adherence rates that are not just a by-product of increased days supply, self-selection, home delivery auto-refill programs, or LIS status.

This study evaluated the overall impact of dispensing channel on adherence to medications in patients with 3 of the most prevalent comorbid conditions among Medicare patients. Given that two thirds of Medicare beneficiaries have at least 2 or more chronic conditions and patients with multiple chronic conditions account for more than $90 \%$ of Medicare health care spend, more studies are warranted to generalize our findings to all Medicare patients with other comorbid conditions, such as ischemic heart disease, stroke, chronic kidney disease, chronic obstructive pulmonary disease, and arthritis, which are also highly prevalent and expensive conditions among Medicare beneficiaries.

This study provides evidence to managed care organizations, health plans, and the Centers for Medicare \& Medicaid Services that home delivery is especially useful to address medication nonadherence among Medicare patients with comorbid conditions of diabetes, hypertension, and high blood cholesterol. Increased adherence among Medicare patients with comorbidities may improve health outcomes as well as reduce the health care spend by Medicare on these beneficiaries, which currently constitutes $90 \%$ of total Medicare spend.

\section{Authors}

REETHI N. IYENGAR, PhD, MBA, MHM, Research and Analytics; ABBEY L. LEFRANCOIS, PharmD, Clinical Program Management, Government Programs; ROCHELLE R. HENDERSON, PhD, MPA, Research and Analytics; and REBECCA M. RABBITT, MS, PharmD, Government Programs, Medicare, Express Scripts Holding Company, St. Louis, Missouri.

AUTHOR CORRESPONDENCE: Reethi lyengar, PhD, MBA, MHM, Research and Analytics, Express Scripts, 4600 N. Hanley Rd., PTIC08, St. Louis, MO 63134. E-mail: rniyengar@express-scripts.com.

\section{ACKNOWLEDGMENTS}

The authors thank Douglas Mager, MA, and Ruth Martinez, RPh, with Express Scripts, for their helpful suggestions in the critical review of the manuscript. The authors acknowledge and thank Sharon Frazee, PhD, from Pharmacy Benefit Management Institute, for her valuable input to the design of this study. The authors also thank the anonymous reviewers who provided valuable suggestions and helped improve the manuscript.

\section{REFERENCES}

1. Centers for Medicare and Medicaid Services. Chronic conditions among Medicare beneficiaries. Chartbook: 2012 ed. Available at: http://www.cms.gov/ Research-Statistics-Data-and-Systems/Statistics-Trends-and-Reports/ChronicConditions/Downloads/2012Chartbook.pdf. Accessed February 17, 2016.

2. American Hospital Association. Trendwatch. Are Medicare patients getting sicker? December 2012. Available at: http://www.aha.org/research/ reports/tw/12dec-tw-ptacuity.pdf. Accessed February 17, 2016.

3. Osterberg L, Blaschke T. Adherence to medication. N Engl J Med. 2005;353(5):487-97.

4. Brunton SA. Improving medication adherence in chronic disease management. J Fam Pract. 2011;60(4 Suppl):S1-S8.

5. McDermott MM, Schmitt B, Wallner E. Impact of medication nonadherence on coronary heart disease outcomes: a critical review. Arch Intern Med. 1997;157(17):1921-29.

6. New England Healthcare Institute. Thinking outside the pillbox: a system-wide approach to improving patient medication adherence for chronic disease. NEHI Research Brief. August 2009. Available at: http://www. nehi.net/writable/publication_files/file/pa_issue_brief_final.pdf. Accessed February 17, 2016.

7. Stuart B, Loh FE, Roberto P, Miller LM. Increasing Medicare Part D enrollment in medication therapy management could improve health and lower costs. Health Aff (Millwood). 2013;32(7):1212-20.

8. Kennedy J, Tuleu I, Mackay K. Unfilled prescriptions of Medicare beneficiaries: prevalence, reasons, and types of medicines prescribed. J Manag Care Pharm. 2008;14(6):553-60. Available at: http://www.amcp.org/data/jmcp/ JMCPMaga_553-560.pdf.

9. Iyengar RN, Henderson RR, Visaria J, Frazee SG. Dispensing channel and medication adherence: evidence across 3 therapy classes. Am J Manag Care. 2013;19(10):798-804

10. Iyengar RN, Balagere DS, Henderson RR, LeFrancois AL, Rabbitt RM, Frazee SG. Association between dispensing channel and medication adherence among Medicare beneficiaries taking medications to treat diabetes, high blood pressure, or high blood cholesterol. J Manag Care Spec Pharm. 2014;20(8):851-61. Available at: http://www.jmcp.org/doi/abs/10.18553/ jmcp.2014.20.8.851

11. Gijsen R, Hoeymans N, Schellevis F, Ruwaard D, Satariano WA, van den Bos GA. Causes and consequences of comorbidity: a review. J Clin Epidemiol. 2001;54(7):661-74.

12. Stuck A, Walthert J, Nikolaus T, Bula C, Hoffman C, Beck J. Risk factors for functional status decline in community-living elderly people: a systematic literature review. Soc Sci Med. 1999;48(4):445-69.

13. Williams J, Steers WN, Ettner SL, Mangione CM, Duru OK. Cost-related nonadherence by medication type among Medicare Part D beneficiaries with diabetes. Med Care. 2013;51(2):193-98.

14. Zhang Y, Baik SH, Lave JR. Effects of Medicare Part D coverage gap on medication adherence. Am J Manag Care. 2013;19(6):e214-24.

15. Zhang L, Zakharyan A, Stockl KM, Harada AS, Curtis BS, Solow BK. Mail-order pharmacy use and medication adherence among Medicare Part D beneficiaries with diabetes. J Med Econ. 2011;14(5):562-67.

16. Duru OK, Schmittdiel JA, Dyer WT, et al. Mail-order pharmacy use and adherence to diabetes-related medications. Am J Manag Care. 2010;16(1):33-40. 


\section{Medication Nonadherence Among Medicare Beneficiaries with Comorbid Chronic Conditions: Influence of Pharmacy Dispensing Channel}

17. Sharma KP, Taylor TN. Pharmacy effect on adherence to antidiabetic medications. Med Care. 2012;50(8):685-91.

18. Carroll NV, Brusilovsky I, York B, Oscar R. Comparison of costs of community and mail service pharmacy. J Am Pharm Assoc. 2005;45(3):336-43.

19. Visaria J, Frazee SG, Devine ST. Asthma controller adherence in mail order pharmacy compared with retail pharmacy. Am J Pharm Benefits. 2012;4(3):e73-80

20. Schmittdiel JA, Karter AJ, Dyer WT, Chan J, Duru OK. Safety and effectiveness of mail order pharmacy use in diabetes. Am J Manag Care. 2013;19(11):882-87.

21. Khandelwal N, Duncan I, Rubinstein E, et al. Medication adherence for 90-day quantities of medication dispensed through retail and mail order pharmacies. Am J Manag Care. 2011;17(11):e427-34.

22. Liberman JN, Girdish C. Recent trends in the dispensing of 90-day supply prescriptions at retail pharmacies: implications for improved convenience and access. Am Health Drug Benefits. 2011;4(2):95-100.

23. Partnership for Solutions JHU. Chronic Conditions and Disability: Analysis of 2000 Census. Baltimore, MD: Partnership for Solutions; 2003.

24. Noyes K, Liu H, Temkin-Greener H. Medicare capitation model, functional status, and multiple comorbidities: model accuracy. Am J Manag Care. 2008;14(10):679-90

25. Krumholz HM. Outcomes research: generating evidence for best practice and policies. Circulation. 2008;118(3):309-18.

26. Brookhart MA, Stürmer T, Glynn RJ, Rassen J, Schneeweiss S. Confounding control in healthcare database research: challenges and potential approaches. Med Care. 2010;48(6 Suppl):S114-20.

27. Janis IB, Nock MK. Behavioral forecasts do not improve the prediction of future behavior: a prospective study of self-injury. J Clin Psychol. 2008;64(10):1164-74. Available at: http://www.hhs.gov/ohrp/policy/ohrpregulations.pdf. Accessed March 25, 2016.
28. Basic HHS Policy for Protection of Human Research Subjects, 45 CFR Part 46 (2009). Available at: http://www.hhs.gov/ohrp/policy/ohrpregulations.pdf. Accessed March 25, 2016.

29. Wu EQ, Johnson S, Beaulieu N, et al. Healthcare resource utilization and costs associated with non-adherence to imatinib treatment in chronic myeloid leukemia patients. Curr Med Res Opin. 2009;26(1):61-69.

30. Yeaw J, Benner JS, Walt JG, Sian S, Smith DB. Comparing adherence and persistence across 6 chronic medication classes. J Manag Care Pharm. 2009;15(9):728-40. Available at: http://www.amcp.org/data/jmcp/728-740.pdf

31. Karve S, Cleves MA, Helm M, Hudson TJ, West DS, Martin BC. An empirical basis for standardizing adherence measures derived from administrative claims data among diabetic patients. Med Care. 2008;46(11):1125-33.

32. U.S. Census Bureau. Metropolitan and micropolitan statistical areas main. Last revised May 6, 2013. Available at: http://www.census.gov/population/metro/. Accessed February 17, 2016.

33. Brookhart MA, Patrick AR, Dormuth C, et al. Adherence to lipid lowering therapy and the use of preventive health services: an investigation of the healthy user effect. Am J Epidemiol. 2007;166(3):348-54.

34. Centers for Medicare \& Medicaid Services. Part C and D performance data. 2013 Part C \& D Medicare star ratings data. Available at: https://www. cms.gov/Medicare/Prescription-Drug-Coverage/PrescriptionDrugCovGenIn/ PerformanceData.html. Accessed March 25, 2016.

35. Reid RO, Deb P, Howell BL, Shrank WH. Association between Medicare Advantage Plan star ratings and enrollment. JAMA. 2013;309(3):267-74.

36. Whaley C, Reed M, Hsu J, Fung V. PS2-18: Functional limitations, home support, and responses to drug costs among Medicare beneficiaries. Clin Med Res. 2013;11(3):159-60. 


\section{APPENDIX A List of Drugs Used in Calculation of PDC}

The GPI, from Medi-Span, is a hierarchical identifier comprising 7 subsets of 2 digits each that provide progressively more detailed information about a drug, specific to treatment indication.

\begin{tabular}{|c|c|}
\hline Treatment Indication & Identifier \\
\hline \multicolumn{2}{|l|}{ Diabetes } \\
\hline Sulfonylureas & $27-20-00-20,27-20-00-27,27-20-00-30,27-20-00-40,27-20-00-50,27-20-00-60,27-99-78-02$ \\
\hline Biguanides & $27-25-00-50,27-99-25-02,27-99-50-02,27-99-70-02,27-99-80-02$ \\
\hline Meglitinide analogues & $27-28-00-40,27-28-00-60$ \\
\hline Alpha-glucosidase inhibitors & $27-50-00-10,27-50-00-50$ \\
\hline Dipeptidyl peptidase-4 (DPP-4) inhibitors & $27-55-00-50,27-55-00-65,27-55-00-70,27-99-30-02$ \\
\hline Insulin sensitizing agents & $27-60-70-50,27-60-70-60$ \\
\hline \multicolumn{2}{|l|}{ Hypertension } \\
\hline Diuretics & $\begin{array}{l}37-20-00-10,37-20-00-20,37-20-00-30,37-20-00-80,37-50-00-10,37-50-00-20,37-50-00-30 \\
37-60-00-10,37-60-00-20,37-60-00-25,37-60-00-40,37-60-00-50,37-60-00-55,37-60-00-60 \\
37-99-00-02\end{array}$ \\
\hline Beta blockers & $\begin{array}{l}33-10-00-10,33-10-00-25,33-10-00-30,33-10-00-40,33-10-00-45,33-10-00-50,33-20-00-10 \\
33-20-00-20,33-20-00-21,33-20-00-22,33-20-00-30,33-20-00-40,33-30-00-07,33-30-00-10 \\
36-99-20-02\end{array}$ \\
\hline Calcium channel blockers & $\begin{array}{l}34-00-00-03,34-00-00-10,34-00-00-13,34-00-00-15,34-00-00-18,34-00-00-20,34-00-00-24, \\
34-00-00-30\end{array}$ \\
\hline $\begin{array}{l}\text { Angiotensin-converting enzyme inhibitors (ACEs)/ } \\
\text { angiotensin II receptor blockers (ARBs)/direct renin } \\
\text { inhibitors }\end{array}$ & $\begin{array}{l}36-10-00-05,36-10-00-10,36-10-00-20,36-10-00-27,36-10-00-30,36-10-00-33,36-10-00-35 \\
36-10-00-40,36-10-00-50,36-10-00-60,36-15-00-10,36-15-00-20,36-15-00-24,36-15-00-30 \\
36-15-00-40,36-15-00-55,36-15-00-70,36-15-00-80,36-17-00-10,36-99-15-02,36-99-18-02 \\
36-99-30-02,36-99-40-02,36-99-45-03,36-99-60-02,36-99-67-02,36-99-68-03\end{array}$ \\
\hline \multicolumn{2}{|l|}{ High blood cholesterol } \\
\hline HMG CoA reductase inhibitors & $\begin{array}{l}39-40-00-10,39-40-00-30,39-40-00-50,39-40-00-58,39-40-00-60,39-40-00-65,39-40-00-75, \\
39-40-99-02,39-99-40-02\end{array}$ \\
\hline
\end{tabular}

\section{APPENDIX B Baseline Characteristics Across Channels for 90 Days Supply Model}

\begin{tabular}{|c|c|c|c|c|}
\hline \multirow{3}{*}{ Number } & \multicolumn{2}{|c|}{ Home Delivery } & \multicolumn{2}{|c|}{ Retail } \\
\hline & \multicolumn{2}{|c|}{2,437} & \multicolumn{2}{|c|}{1,720} \\
\hline & \multicolumn{4}{|c|}{ Mean (SD) } \\
\hline Age, years & 73.2 & $(6.0)$ & 73.1 & $(6.1)^{\mathrm{a}}$ \\
\hline OOP costs per 30-day adjusted prescriptions for antidiabetics & 10.16 & $(13.88)$ & 6.32 & $(9.51)^{\mathrm{a}}$ \\
\hline OOP costs per 30-day adjusted prescriptions for antihypertensives & 7.87 & $(8.85)$ & 5.52 & $(6.20)^{\mathrm{a}}$ \\
\hline OOP costs per 30-day adjusted prescriptions for antihyperlipidemics & 10.93 & $(13.31)$ & 6.71 & $(8.27)^{\mathrm{a}}$ \\
\hline Disease burden proxy ${ }^{b}$ & 1.8 & $(2.2)$ & 3.6 & $(3.5)^{\mathrm{a}}$ \\
\hline Severity of illness proxy for diabetes ${ }^{c}$ & 1.5 & $(0.7)$ & 1.4 & $(0.6)^{\mathrm{a}}$ \\
\hline \multirow[t]{2}{*}{ Severity of illness proxy for hypertension ${ }^{\mathrm{c}}$} & 1.9 & $(0.9)$ & 2.0 & $(0.9)^{\mathrm{a}}$ \\
\hline & \multicolumn{4}{|c|}{ n (\%) } \\
\hline Baseline adherent for antidiabetics & 2,114 & $(86.8)$ & 1,450 & $(84.3)^{a}$ \\
\hline Baseline adherent for antihypertensives & 2,185 & $(89.7)$ & 1,486 & $(86.4)^{\mathrm{a}}$ \\
\hline Baseline adherent for antihyperlipidemics & 2,231 & $(91.6)$ & 1,505 & $(87.5)^{\mathrm{a}}$ \\
\hline Female & 1,031 & $(42.3)$ & 833 & $(48.4)^{\mathrm{a}}$ \\
\hline Urbanicity & 2,310 & $(94.8)$ & 1,618 & $(94.1)^{\mathrm{a}}$ \\
\hline LIS beneficiaries & 50 & $(2.1)$ & 313 & $(18.2)^{\mathrm{a}}$ \\
\hline
\end{tabular}

a Significantly different from home delivery at $P<0.05$.

bPatient overall disease burden was defined as the number of unique 2-digit GPIs (except diabetes, hypertension, and high blood cholesterol), which indicate distinct drug therapy classes used by the patient beyond the 3 therapy classes.

cSeverity of illness is defined by the number of unique drug groups for which the patient had a prescription claim in 2012 for diabetes and hypertension. GPI = Generic Product Identifier; LIS=low-income subsidy; OOP=out of pocket; SD= standard deviation. 